\section{Respuesta de la OPS al peligro de las enfermedades infecciosas emergentes y reemergentes ${ }^{1}$}

${ }^{1}$ Basado en "Enfermedades infecciosas emergentes y reemergentes y resistencia a los antimicrobianos", tema tratado en la 41. ${ }^{\text {a }}$ Reunión del Consejo Directivo de la Organización Panamericana de la Salud, celebrada en San Juan, Puerto Rico, en septiembre de 1999.
Pese a los enormes adelantos que ha habido en años recientes en el control de las enfermedades infecciosas, estas siguen planteando una grave amenaza para los países de la Región. Algunas, como la infección por el virus de la inmunodeficiencia humana (VIH), la enfermedad de Lyme y las ocasionadas por hantavirus, son de aparición reciente y se suman a otras enfermedades que la humanidad ya conocía pero que emergen de nuevo, tras un período prolongado de inactividad. Este fenómeno se debe, en parte, a la mutación de los microorganismos causales y a la consiguiente aparición de resistencia contra los fármacos habitualmente usados para su tratamiento y control. Para hacer frente a esta situación, en 1995 se elaboró un Plan de Acción Regional cuya finalidad era proporcionar orientación a los Estados Miembros de la Organización Panamericana de la Salud (OPS) sobre la manera de reconocer, prevenir y responder a la amenaza planteada por este tipo de enfermedades.

El Comité Ejecutivo de la OPS, en su 124. a sesión, examinó la estrategia propuesta y la encontró adecuada. A raíz de ello, el 41. ${ }^{\text {er }}$ Consejo Directivo, reunido en septiembre de 1999, aprobó una resolución al respecto cuyo contenido esencial se resume a continuación.

\section{SITUACIÓN EPIDEMIOLÓGICA ACTUAL}

Las enfermedades infecciosas emergentes, según lo establecido por el Instituto de Medicina de los Estados Unidos de América, son aquellas cuya incidencia ha aumentado en los seres humanos en los últimos 20 años. Las reemergentes son, por otra parte, las que han vuelto a aparecer tras una larga y sostenida disminución de su incidencia. De las primeras es ejemplo muy patente el síndrome de inmunodeficiencia adquirida (sida), enfermedad de origen vírico desconocida hasta principios de los años ochenta; de las segundas, el dengue, también vírico, que reapareció con gran ímpetu en los años ochenta, pese a la erradicación del vector de casi toda América Latina y a la consiguiente reducción de su incidencia en los dos decenios anteriores. En los últimos 20 años, el dengue ha recrudecido con vehemencia y actualmente es endémico en casi todo el continente americano, donde en 1998 se notificaron 770000 casos de la enfermedad. 
Algunas epidemias - tomemos como ejemplos la del virus Ebola en el África, la de la peste en la India y, en nuestro continente, la de la insuficiencia pulmonar del adulto causada por un hantavirus (el virus innominado) - son ampliamente divulgadas por la prensa; otras, como la del cólera, enfermedad que se difundió por la Región en 1993 tras un siglo de ausencia, reciben escasa atención. Sucedió algo similar cuando se produjo en 1992 en el Perú una larga epidemia de peste bubónica que había afectado a 2000 personas y causado 90 defunciones.

Entre las principales enfermedades emergentes y reemergentes que son actualmente motivo de alarma en la Región figuran la enfermedad de Lyme, la diarrea por criptosporidiosis y la afección causada por Escherichia coli 0157:H7 en los Estados Unidos; el dengue y la fiebre amarilla en el Brasil; la malaria farmacorresistente por Plasmodium falciparum en algunas zonas de la cuenca amazónica y las infecciones por hantavirus en el Cono Sur. Y en este panorama también suscita gran preocupación la resistencia generalizada de varias especies bacterianas a los antibióticos, como en el caso del agente causal de la tuberculosis.

\section{RESPUESTA DE LA OPS A LA AMENAZA}

Varios países industrializados han hecho frente a la aparición de enfermedades emergentes y reemergentes elaborando nuevos métodos de laboratorio para identificar los microorganismos que las causan, pero aún queda mucho por hacer en cuanto a mejorar las técnicas de diagnóstico y difundir su uso, especialmente en los países en desarrollo. Con el fin de proporcionar a sus Estados Miembros orientación que los ayude a aplicar medidas regionales y subregionales para la prevención y el control de las enfermedades infecciosas emergentes y reemergentes, la OPS ha encaminado su Plan de Acción Regional a lograr las siguientes metas:

- fortalecer las redes regionales de vigilancia de las enfermedades infecciosas emergentes y reemergentes en las Américas;

- establecer infraestructuras nacionales y regionales para dar rápido aviso y pronta respuesta ante la amenaza de dichas enfermedades mediante un mejor sistema de laboratorios y programas de adiestramiento multidisciplinarios para profesionales;

- impulsar la investigación en materia de diagnóstico rápido, epidemiología y prevención;

- fortalecer en la Región la adopción de estrategias eficaces de prevención y control.
En 1996-1998 se convocó un Grupo de Estudio sobre la Vigilancia de las Enfermedades Infecciosas Emergentes y Reemergentes integrado por expertos de varios países de la Región y de la Organización Mundial de la Salud (OMS), el cual formuló directrices para poner en marcha el Plan Regional de Acción para Combatir las Enfermedades Infecciosas Emergentes y Reemergentes elaborado por la OPS. Atendiendo a las recomendaciones de este Grupo, la OPS organizó reuniones subregionales donde se establecieron las bases para un sistema de vigilancia que permitiera monitorear la resistencia a los antimicrobianos, notificar la aparición de sucesos epidemiológicos alarmantes en el momento mismo en que estuvieran sucediendo, y aportar retroinformación continua y apoyo inmediato.

\section{Estrategia y cooperación técnica de la OPS}

Las actividades de cooperación técnica de la OPS han tenido como finalidad alcanzar las metas del Plan de Acción Regional y también se han guiado por las recomendaciones del Grupo de Estudio, que se reúne anualmente para analizar la situación y formular sugerencias para la cooperación técnica futura. En su esfuerzo por hacer frente a tan díficil situación, la OPS ha adoptado un enfoque triple que consiste en los siguientes componentes:

- la vigilancia de las enfermedades infecciosas emergentes, incluidas la creación y puesta en marcha de una plataforma electrónica para comunicar de manera instantánea la aparición de las enfermedades;

- la detección de brotes y la respuesta inmediata para controlarlos;

- la vigilancia y prevención de la resistencia a los antimicrobianos.

Las actividades de la OPS orientadas a fortalecer los laboratorios ${ }^{2}$ tendrán un efecto decisivo en el control de las enfermedades emergentes y reemergentes.

\section{Actividades de vigilancia}

Las enfermedades transmisibles siguen constituyendo las principales causas de morbilidad y mortalidad en los países en desarrollo, donde producen pérdidas económicas enormes por su efecto

${ }^{2}$ Estas actividades están a cargo del Programa de Enfermedades Transmisibles de la División de Prevención y Control de Enfermedades y del Programa de Medicamentos Esenciales y Tecnología de la División de Desarrollo de Sistemas y Servicios de Salud. 
sobre el turismo y la exportación de alimentos, aunque también representan un peligro para los países industrializados. La gravedad del problema exige una respuesta concertada a escala regional, puesto que los países no se ven afectados aisladamente. Debido al enorme aumento de los viajes internacionales, entre otros factores, la propagación de las enfermedades se puede producir en cuestión de horas en zonas que no se veían afectadas. Como consecuencia de esta situación, todas las actividades de la OPS, con pocas excepciones, tienen un enfoque subregional y dependen de la cooperación técnica entre los países. Hasta ahora se han implantado, en la región amazónica y en el Cono Sur, redes subregionales de laboratorios para la vigilancia de las enfermedades infecciosas emergentes, con la colaboración de los Centros para el Control y la Prevención de Enfermedades (CDC) de los Estados Unidos, y se está contemplando la creación de una red similar en Centroamérica.

En 1998 la OPS patrocinó un proyecto de cooperación técnica entre Argentina y Chile para el diagnóstico, la vigilancia y la investigación de las enfermedades producidas por hantavirus. Actividades afines en otros países de la Región también han recibido apoyo, siendo un ejemplo concreto el aporte de reactivos por la Argentina para el diagnóstico de infecciones por hantavirus. Asimismo, la OPS colabora con varias entidades, entre ellas diversos institutos nacionales de investigación, ministerios de salud y laboratorios nacionales de referencia, para crear un sistema regional para la vigilancia de las enfermedades infecciosas y fortalecer los programas existentes para la vigilancia de la resistencia de determinados agentes patógenos a los antimicrobianos. El fortalecimiento de la vigilancia de las enfermedades se basará en actividades más eficientes de recopilación y análisis de datos, investigación y rápida intervención en situaciones de peligro. Sus objetivos son 1) fortalecer la puesta en práctica de estrategias eficaces de prevención, control y vigilancia; 2) crear una infraestructura nacional y regional para el aviso inmediato de situaciones de amenaza y la rápida respuesta frente a ellas.

El nuevo sistema de vigilancia utilizará la Internet para crear sistemas de correo electrónico y la Intranet (acceso cerrado) para establecer comunicaciones rápidas y seguras con los ministerios de salud, las representaciones de la OPS/OMS, instituciones nacionales de investigación y otras entidades interesadas. Los datos validados se podrán consultar en el sitio de la Web que pertenece a la OPS y todos los participantes tendrán igual acceso a la base de datos común para su análisis. Una vez que se hayan establecido los sistemas de vigilancia y la infraestructura correspondientes, se hará un monitoreo continuo de los agentes patógenos y las enfer- medades infecciosas emergentes, con especial atención a la detección temprana de brotes y epidemias; se evaluarán las repercusiones socioeconómicas y sanitarias de la situación y su probable evolución; se determinará la capacidad de respuesta en el ámbito local; se identificarán las medidas de control más eficaces y se evaluarán otras necesidades inmediatas. En todo este contexto tiene una importancia capital la definición de casos desde el punto de vista de la validación de datos y su comparación.

\section{Detección de brotes y respuestas ante ellos}

El concepto de vigilancia debe ampliarse para incluir no solo la recaudación de datos epidemiológicos, sino también la planificación de intervenciones y la integración de actividades por los sectores público y privado encaminadas a afrontar los brotes de enfermedades infecciosas. Esta estrategia programática es la que sigue la OPS en el momento actual. Para complementarla y para que los países puedan responder con eficiencia y eficacia a la amenaza de brotes de enfermedad, especialmente después de situaciones de desastre, la OPS viene trabajando con varios países, primero en Centroamérica, en la creación y fortalecimiento de equipos nacionales multidisciplinarios. El enfoque actual se centra en una estrategia prolongada y sustentable orientada a formar, en cada país de la Región, profesionales capacitados y provistos de los instrumentos y otros medios necesarios para dirigir la respuesta nacional a los brotes epidémicos y otras situaciones de peligro para la salud pública.

\section{La resistencia a los antimicrobianos}

La farmacorresistencia de algunos microorganismos, que en determinados casos se intensifica paulatinamente, menoscaba gravemente la lucha contra enfermedades tan devastadoras como la tuberculosis, el paludismo, las enfermedades diarreicas, el cólera y la neumonía, que ocasionan, en su conjunto, 10 millones de defunciones anuales en el mundo. A ello se añade la lentitud con la que se están elaborando medicamentos alternos para reemplazar a los que han perdido su eficacia. Este aumento de la resistencia a los antimicrobianos se observa más patentemente en el caso de Streptococcus pneumoniae, principal agente causal de infecciones respiratorias agudas mortales en los niños. El tipo de resistencia que se observa en los hospitales de toda la Región podría dejar al personal médico y de salud pública completamente desprovisto de los medios para la prevención y el tratamiento de estas infecciones. Las bacterias resistentes a los antibióti- 
cos causan alrededor de $60 \%$ de las infecciones nosocomiales en los Estados Unidos y, como resultado, las personas permanecen hospitalizadas más tiempo y corren un mayor riesgo de morir.

El uso irracional o inapropiado de los antibióticos ha sido el factor determinante en el surgimiento de cepas bacterianas resistentes. Por lo tanto, se impone la necesidad de evitar la prescripción indebida de estos medicamentos por parte de los médicos, y su consumo sin prescripción por la población en general. Asimismo, es necesario establecer normas legales y políticas que exijan el uso racional de los antibióticos.

Plantea una dificultad adicional la ausencia de datos fidedignos sobre la verdadera magnitud de la resistencia a los antimicrobianos en la Región, sin los cuales se dificulta la planificación de intervenciones. A fin de encarar esta situación y el uso indebido de los antibióticos, la OPS empieza a trabajar muy de cerca con expertos y organizaciones en los países a fin de promover, en un futuro próximo, los cambios necesarios en el ámbito de la política sanitaria. Los CDC, por otra parte, han proporcionado adiestramiento en el uso del Sistema de Información de Laboratorios de Salud Pública a profesionales de diversos países caribeños de habla inglesa.

La OPS, junto con la OMS, el Ministerio de Sanidad y Asistencia Social de Venezuela y la Sociedad Panamericana de Infectología, patrocinó la Conferencia Panamericana de Resistencia Antimicrobiana, donde se recopiló información sobre la situación actual de la farmacorresistencia en la Región. En esa ocasión se formularon varias recomendaciones que se incorporarán a un plan trienal cuyos objetivos son 1) fortalecer la vigilancia; 2) fomentar la formulación de políticas; 3 ) estimular la participación de la industria farmacéutica en las actividades estratégicas; 4) vigilar el uso de antibióticos en la cría de animales, y 5) promover la prescripción y el uso adecuados de estos medicamentos. Desde 1996, la OPS, junto con el Centro de Laboratorios para el Control de Enfermedades (Laboratory Center for Disease Control, LCDC) del Canadá, ha colaborado con los países para fortalecer su infraestructura y capacidad técnica de laboratorio y epidemiología para la vigilancia de los agentes patógenos entéricos de interés.

\section{FUTURAS ACTIVIDADES DE COOPERACIÓN TÉCNICA}

\section{Actividades de vigilancia}

Las actividades que consisten en la difusión de información continuarán con la publicación de informes técnicos en papel y en formato electrónico.
Una vez que esté en funcionamiento la plataforma electrónica para los sistemas de notificación de enfermedades infecciosas emergentes, los datos notificados se colocarán en ella, al alcance de todos los interesados.

Los buenos resultados arrojados por el establecimiento de las dos redes subregionales de laboratorio (la amazónica y la del Cono Sur) han llevado a sondear la posibilidad de una iniciativa similar en Centroamérica, como se señaló anteriormente. Ello implica trabajar con los países para determinar las prioridades, necesidades de laboratorio y opciones de financiamiento.

La OPS, mediante su Programa de Enfermedades Transmisibles, seguirá manteniendo un sistema de alarma epidemiológica inmediata entre los países y compartiendo información relativa a las enfermedades emergentes y reemergentes, incluidos los informes de brotes epidémicos. También se promoverá la aplicación de definiciones de casos estandarizados para que la información proporcionada por los países al sistema regional sea comparable. La OPS seguirá trabajando con la OMS a fin de someter a pruebas piloto el Reglamento Sanitario Internacional, y seguirá prestando asistencia a los países para que adquieran los reactivos necesarios para el diagnóstico rápido de las enfermedades.

\section{Detección de brotes y respuesta ante ellos}

Otras actividades en curso son la adaptación de materiales didácticos sobre la investigación de brotes y la respuesta adecuada cuando se producen, así como el fortalecimiento de la capacidad de los laboratorios para diagnosticar los agentes causales de las enfermedades emergentes y reemergentes. En determinados países se evaluará la integridad de los sistemas nacionales de vigilancia, especialmente desde el punto de vista de su capacidad para detectar brotes y responder a ellos con rapidez.

Las actividades de adiestramiento, como ya se ha señalado, se dirigirán a equipos multidisciplinarios de seis personas para cada país elegido. Habrá en cada equipo un epidemiólogo, un laboratorista, un profesional de la enfermería, un especialista en desastres, un representante de sanidad de las fuerzas armadas y un experto en comunicación social. Estos individuos serán adiestrados en cursos subregionales al final de los cuales se les entregarán laboratorios de campo, reactivos y otros insumos que les permitan reaccionar debidamente en caso de un brote de enfermedad. En cada país también se fomentará la capacitación de instructores para garantizar la sustentabilidad del proyecto. Por lo tanto, se proporcionará apoyo a los equipos nacionales de tal modo que ellos mismos se ocupen de llevar a cabo 
la capacitación necesaria en los niveles nacional, provincial y local.

\section{La resistencia a los antimicrobianos}

Se perpetuará la colaboración de la OPS con el LCDC, a fin de apoyar la vigilancia continua de la resistencia a los antibióticos, particularmente de Shigella, Salmonella y Vibrio cholera spp. Estas actividades de vigilancia complementarán las de la OMS, que se dirigen a una gama más extensa de agentes patógenos y que se realizan principalmente en hospitales. También se emprenderá el Plan Regional de Acción ya mencionado, cuyos dos componentes principales son 1) el fortalecimiento de la capacidad para determinar la magnitud y consecuencias de la resistencia a los antibióticos y aumentar la aplicación de medidas para enfrentar el problema. Entre estas últimas figuran el acopio de datos sobre políticas nacionales, reglamentos y normas; la elaboración de métodos para promover el uso racional de los medicamentos antimicrobianos y la sensibilización de los profesionales de la salud, las autoridades sanitarias y el público en general en torno al riesgo que entrañan el uso irracional de los antibióticos y el consiguiente aumento de la resistencia a estos fármacos. A fin de llevar a cabo estas y muchas otras de las actividades programadas, se torna necesaria la ayuda de otros organismos de cooperación multinacional, bilateral o privados. Será tarea de la OPS dirigir en la Región la coordinación de dichas actividades y proporcionar el apoyo técnico necesario para su cumplimiento.

\section{SYNOPSIS}

\section{PAHO's response to the threat of emerging and reemerging communicable diseases}

Currently, emerging and reemerging infectious diseases are among the most challenging health problems being faced by countries of the Region. Some of these diseases have appeared in recent times; others have afflicted humanity in the past and are now reappearing, with renewed strength, in some cases as a result of the inadequate or unnecessary use of antibiotics on the part of the public at large and even of medical professionals. In light of the danger posed by these diseases, especially in poor countries where there are scant infrastructural and economic resources with which to combat them, in 1995 PAHO adopted a Regional Plan of Action and established a Task Force for the Surveillance of Emerging and Reemerging Infectious Diseases, with the aim of establishing effective and sustainable mechanisms for disease surveillance, diagnosis, prevention, and control in the entire American continent. The overall thrust of these measures and of the technical cooperation PAHO provides to the different countries is the subject of this report. 